\title{
The design of useful graphics in Bertin's thought. A case study from the Urban Atlas of Jeddah.
}

\author{
Mohsen DHIEB ${ }^{\mathrm{a},} *$, Abdelhamid JAMIL ${ }^{\mathrm{b}}$ and Ahmed MOHARREM ${ }^{\mathrm{c}}$ \\ ${ }^{a}$ King Abdulaziz University of Jeddah - Faculty of Arts and Humanities - Department of Geography and GIS. \\ Mohsendhieb2003@yahoo.fr \\ ${ }^{b}$ King Abdulaziz University of Jeddah - Faculty of Arts and Humanities - Department of Geography and GIS. \\ Abdlhamedjamil@gmail.com \\ ${ }^{c}$ King Abdulaziz University of Jeddah - Faculty of Arts and Humanities - Department of Geography and GIS. \\ Abdlhamedjamil@gmail.com
}

* Corresponding author: Mohsen DHIEB

Keywords Graphics Design- Semiology of Graphics- Bertin's rules - Urban Atlas of Jeddah.

\begin{abstract}
:
The Semiology of Graphics of French cartographer J. Bertin is not only a seminal work on maps design, it contains also very useful sets of rules and insights that apply to graphics (BERTIN, 1970, 1973, 1987, 2005; DHIEB, 2018). Even though the visual variables are considered by cartographers as one of the most principal discoveries of the book (DE GOLBERY et al., (1996; PALSKY, 2017;), Bertin stated very useful techniques, innovations, artefacts and examples that apply specifically to the design of maps and graphics.
\end{abstract}

One of the most important issues that were not discussed considering the visual variables and the overall design addresses the design of graphics and the use of visual variables. When handling statistical data to design graphics, these latter should not be built randomly; their design should obey strict rules and contain necessary components with regards to the data handled. May we talk about a kind of guideline to help users to choose the most efficient type of graphic depending on the data characteristics the design context, and the message to convey?

Yet the specialized software dedicated to graphics design such as Excel do not answer this question and do not help sufficiently the map users to make the best choice and when a choice is made, a useful explanation is not furnished to justify this choice by common users or by cartographers. Our hypothesis is that the design of graphics and the choice of appropriate visual variables according to their properties is worth it: it may greatly influence their attractivity and increase their readability and memorization.

This issue was encountered by authors when designing the Urban Atlas of Jeddah (UAJ). Besides detailed maps that portray the spatial distribution and analysis of various data on Jeddah and its districts, the UAJ needed that some of these data to be converted into graphics when the focus is on attribute characteristics rather than on spatial distribution. When inserted in one Atlas, the choice of one given type of graphics does not depends only on scientific criteria but also on design considerations (choice of appropriate visual variables, consistency, homogeneity, and relevance...). For instance, we should not put in one single atlas only one type of graphics, nor the same looks in terms of retained visual variables.

Yet, the recent Arabic translation of the Semiology of Graphics (DHIEB, 2018) gave them very useful guidelines in designing Atlas graphics. The goal of this paper is first to put altogether Bertin's principles and insights that should apply to the design of graphics; second to implement such guidelines in the UAJ project; and third to evaluate such uses stated 50 years ago by Bertin and other graphicians (BERTIN, 1970, 1973, 1987, 2005; BONIN, 1983; BONIN S. and BONIN M., 1989; GIMENO, 1980; BORD, 2000) with regards to the scientific and technical evolution of graphics design. Full automation of graphical design (GRONOFF, J.-D., 1983; MULLER, J.C., 1984) should be inspired by Bertin's statements on the use of visual variables and the multiple rules that should be extracted from the Graphics Design Guideline (GDG) developed by the authors. Examples of simple graphics or of graphics processing (AMADO, 1994) from the UAJ are furnished according to the sets of prototypes of graphics realized. 